BULL. AUSTRAL. MATH. SOC.

VOL. 26 ( 1982$), 477-478$.

\title{
LINEAR GROUPS: ON NON-CONGRUENCE SUBGROUPS AND PRESENTATIONS
}

\section{Phillip Ronald Helm}

The central theme of this thesis is the calculation of explicit presentations for certain linear groups both for their intrinsic interest and for their use in finding non-congruence subgroups in $\mathrm{SL}_{2}$ of quadratic imaginary number fields.

Let $\operatorname{GL}(n, R)$ denote the group of $n \times n$ invertible matrices with entries from a ring $R$ with unity. When $R$ is commutative $\operatorname{SL}(n, R)$ is the subgroup of $\operatorname{GL}(n, R)$ consisting of matrices with determinant 1 .

Also let $\mathbb{Z}\left(\omega_{d}\right)$ be the ring of imaginary quadratic integers of the form $a+b \omega_{d} ; a, b \in \mathbb{Z}$ and $\omega_{d}=\sqrt{ } d$ if $d \neq 1 \bmod 4$, otherwise $\omega_{d}=\frac{1}{2}(1+d)$, with $0>d \in \mathbb{Z}$. When $d$ has no square factors $\mathbb{Z}\left(\omega_{d}\right)$ is the full ring of integers in the imaginary quadratic number field of discriminant $d$. Let $\mathbb{Z}_{n}\left(\omega_{d}\right)$ be $\mathbb{Z}\left(\omega_{d}\right)$ factored by the principal ideal generated by $n \in \mathbb{Z}$.

The first chapter of the thesis is a computation of the simple factor groups in the composition series of $\operatorname{SL}\left(m, \mathbb{Z}_{n}\left(\omega_{d}\right)\right)$. Using results from the first chapter the second gives a solution of the 'congruence subgroup problem' for $\mathrm{SL}\left(2, \mathbb{Z}\left(\omega_{d}\right)\right.$ ) (which is an alternative one to Serre's) by giving examples of non-congruence subgroups.

The third and fourth chapters deal with presentations of certain $\operatorname{GL}(n, R)$ and $\operatorname{SL}(n, R)$. Early in Chapter Three the theorem used to give

Received 2 September 1982. Thesis submitted to La Trobe University, March 1982. Degree approved July 1982. Supervisor: Dr G.E. Davis. 
results in Chapter Four is developed, and using this a simple presentation of $\operatorname{SL}\left(2, \mathbb{Z}_{p}\right)$ is calculated.

Chapter Four, based on Chapter Three, gives a presentation of $\mathrm{GL}(n, L)$ for various $n$, when $\mathrm{L}$ is a certain type of ring of linear operators. Let $A$ be an ideal of the ring of bounded linear operators mapping a Banach space (over a field $F$ ) into itself and let $L=\{A+\lambda I \mid A \in A, \lambda \in F\}$. If the resolvent of each operator of $A$ is dense in $F$ then $L$ is universal for $G E(2)$. If $F$ is either $\mathbb{R}$ or $\mathbb{C}$ and $A$ is the ideal of operators with finite dimensional range then $L$ is a universal $\mathrm{GE}(n)$-ring.

\section{References}

[1] P.M. Cohn, "On the structure of the $\mathrm{GL}_{2}$ of a ring", Inst. Houtes Études Sci. Puỏz. Math. 30 (1966), 365-413.

[2] Fritz J. Grunewald and Joachim Schwermer, "Free non-abelian quotients of $\mathrm{SI}_{2}$ over orders of imaginary quadratic numberfields", $J$. Algebra 69 (1981), 298-304.

[3] P.R. Helm, "A presentation for $\operatorname{SL}\left(2, \mathbb{Z}_{p}\right)$ ", Comm. Algebra (to appear).

[4] P.R. Helm, "Generators and relations for certain linear groups over rings of linear operators", Comm. Algebra (to appear).

[5] Jean-Pierre Serre, "Le problème des groupes de congruence pour $\mathrm{SL}_{2}$ ", Ann. of Math. (2) 92 (1970), 489-527.

[6] Richard G. Swan, "Generators and relations for certain special linear groups", Adv. in Math. 6 (1971), 1-77.

[7] Hans J. Zassenhaus, "A presentation of the groups PSL $(2, p)$ with three defining relations", Canad. J. Math. 21 (1969), 310-311.

3/22 Gibbs Street,

Balaclava,

Victoria 3183, Australia. 